\title{
Fibroids and Infertility
}

\author{
${ }^{1}$ Harpreet Kaur, ${ }^{2}$ Kamini A Rao
}

\begin{abstract}
Fibroids are the commonest benign tumors of female genital tract. Though fibroids may not be a sole cause for infertility in majority of cases, but it has been seen that there is an improvement in pregnancy rates after myomectomy. Fibroids that distort the uterine cavity and large intramural fibroids are shown to be associated with adverse effects on reproductive function. Available evidence suggests that submucosal, intramural, and subserosal fibroids interfere with fertility in decreasing order of importance. In infertile women and those with recurrent pregnancy loss, myomectomy should be considered only after a thorough evaluation has been completed. Medical management of fibroids has no role in treatment of fertility rather it might lead to delay in the final treatment for infertility. Preoperative medical treatment with a $\mathrm{GnRH}$ agonist should be considered for women who are anemic and those undergoing hysteroscopic myomectomy. Subserosal fibroids have least effect on fertility, so they do not need removal before infertility treatment.
\end{abstract}

Keywords: Fibroids, Infertility, Myomectomy, In vitro fertilization.

Keymessage: Fibroids are important cause of infertility; Removal of submucousal fibroids is warranted before IVF.

Search methodology: Data were sourced from the electronic database PubMed, MEDLINE, OVID, Cochrane Database of systematic reviews and published guidelines on fibroids and infertility. Abstracts from papers and posters presented at the international meetings, published and unpublished studies, and expert opinion was considered.

How to cite this article: Kaur H, Rao KA. Fibroids and infertility. Int J Infertil Fetal Med 2014;5(1):1-7.

Source of support: Nil

Conflict of interest: None

Date of Received: 30-03-14

Date of Acceptance: 20-04-14

Date of Publication: April 2014

\section{INTRODUCTION}

Fibroids are one of the most common benign tumors of the female genital tract. Fibroids can be seen in almost 20 to $40 \%$ of women in reproductive age group. In majority of cases they are asymptomatic. ${ }^{1}$ Symptoms when present are

\footnotetext{
${ }^{1}$ Assistant Professor, ${ }^{2}$ Medical Director

${ }^{1}$ Department of Obstetrics and Gynecology, Gian Sagar Medical College and Hospital, Chandigarh, India

${ }^{2}$ Department of Reproductive Medicine, Bangalore Assisted Conception Centre, Bengaluru, Karnataka, India

Corresponding Author: Harpreet Kaur, Assistant Professor Department of Obstetrics and Gynecology, Gian Sagar Medical College and Hospital, Chandigarh, India, Phone: 09872221818 e-mail: drharpreet_sidhu@hotmail.com
}

mainly related to menstrual abnormalities, mass effect, and sometimes infertility and pregnancy loss. Though fibroids may not be direct cause of infertility in majority of cases, but it has been seen that there is an improvement in pregnancy rates after myomectomy. The adverse effects of fibroids on fertility are mainly related to their location-the worse effects are seen with submucosal fibroids and subserosal have minimal effect on fertility. Most myomas are asymptomatic, are noted incidentally on clinical examination or on ultrasound examination performed for other indications, and do not require treatment. ${ }^{1,2}$ Symptoms or complaints that require evaluation and possible therapy can be categorized into four groups: gynecologic indications for abnormal uterine bleeding, pelvic pain, or lower urinary tract symptoms; reproductive disorders, such as infertility and repeated pregnancy loss; pregnancy-related indications both prior to conception and during pregnancy; and prior to in vitro fertilization (IVF) depending on myoma size and location. ${ }^{2,3}$

\section{ETIOLOGY AND PATHOPHYSIOLOGY OF FIBROIDS}

Myomas increase in size during the reproductive years and regress at menopause. This relationship suggests regulation by cyclic secretion of estradiol, progesterone, or both, or by factors related to ovarian steroid secretion, for growth and development. The exact cause of these tumors is unknown. Though the entire myometrium is exposed to the same hormonal milieu, a single cell line demonstrates a differential sensitivity and enhanced though restricted growth. What contributes to this enhanced and varied sensitivity and growth patterns on the myometrium, not only from patient to patient but also within the same uterus, is unknown. Fibroids arise from genetic alterations in a single myometrial cell and thus often are described as clonal. ${ }^{1-3}$ Factors that influence myoma growth include genetic predisposition and chromosomal differences in the tumors, hormonal dependency (specifically ovarian steroids), and interaction with insulin and a variety of peptide growth factors.

Myomas may be single, multiple, and of varying size. The macroscopic appearance is that of a firm, round tumor with a surrounding pseudocapsule. The microscopic appearance of the tumors differs considerably depending on the degree of interlacing smooth muscle fibers and fibrous tissue and the degree of calcification and degeneration. ${ }^{2}$ 


\section{MYOMAS AND REPRODUCTIVE FUNCTION}

Though fibroids may not be direct cause of infertility in majority of cases, but 2 to $3 \%$ cases of infertility may be attributed to the effects of myomas when all other causes are excluded. . $^{1,2,4}$

Various mechanisms by which myomas lead to infertility may be described as follows:

- Anovulatory cycles occur more commonly in presence of fibroids.

- Displacement of the cervix that may reduce exposure to sperm.

- Enlargement or deformity of the uterine cavity that may interfere with sperm migration and transport.

- Obstruction of the proximal fallopian tubes.

- Altered tuboovarian anatomy, interfering with ovum capture.

- Interference with prostaglandin-induced uterine contractions, which are thought to enhance sperm migration.

- Endometrial changes (atrophy, ulceration, focal hyperplasia, and polyps), vascular alterations (venous congestion, venule ectasia, and impaired blood flow) in the endometrium.

- Endometrial inflammation or secretion of vasoactive substances.

Fibroids may not be the sole cause for infertility. Quite often, an infertile patient with uterine leiomyomata is found to have some other cause of infertility. It is very important to investigate all other causes of infertility in both the partners before considering removal of myoma for treatment of infertility. The ultimate decision regarding disposal of the tumors depends on their size and location. Usually, small subserous leiomyomata are not considered a factor leading to infertility. But leiomyomata which are intramural or submucous and of significant size, may well be factors causing the infertility, and a myomectomy may be rewarded with a subsequent pregnancy. ${ }^{1,2,4}$

\section{EFFECT OF FIBROIDS ON PREGNANCY}

Uterine leiomyomata are associated with a significantly increased risk of spontaneous abortion. Various mechanisms have been proposed to explain the occurrence of spontaneous abortion from uteri with leiomyomata. These include disturbances in uterine blood flow, alterations in blood supply to the endometrium, uterine irritability, rapid growth or degeneration of leiomyomata during pregnancy, difficulty in enlargement of the uterine cavity to accommodate for the growth of the fetus and placenta, and interference with proper implantation and placental growth by poorly developed endometrium or by subjacent leiomyomata. Implantation in a thin, poorly vascularized endometrium over a submucous leiomyoma is doomed to failure, because proper growth and development of the embryo and placenta are impossible. ${ }^{1}$ Ben-Nagi et al ${ }^{5}$ studied the effect of submucosal fibroids in implantation. Women with submucous fibroids were shown to have significantly lower concentrations of glycodelin and interleukin-10 in midluteal phase uterine flushings, emphasizing the association of submucosal fibroids with adverse reproductive outcome. ${ }^{5}$ Buttram and Reiter ${ }^{6}$ reported that spontaneous abortion rate of $41 \%$ in the presence of myoma which was reduced to $19 \%$ after myomectomy.

In a systematic review of effect of fibroids on reproductive outcome, myomas have been reported to increase the risk of malpresentations (odds ratio (OR) 2.9; 95\% confidence interval (CI), 2.6-3.2), cesarean section (OR, 3.7; 95\% CI, 3.5-3.9), preterm delivery (OR, 1.5; 95\% CI, 1.3-1.7), and spontaneous miscarriage (OR, 1.6; 95\% CI, 1.3-2.0). ${ }^{7}$

Location of fibroids is important as far as adverse pregnancy outcome is concerned. It has been seen that fibroids adjacent to the placental site have been associated with adverse pregnancy outcome like increased risks for bleeding, abruption, and premature rupture of membranes. ${ }^{8}{ }^{89}$ In a study to analyze the effect of fibroids in patients with recurrent pregnancy loss by Saravelos et al, ${ }^{10}$ it was shown that resection of fibroids distorting the cavity leads to almost doubling of live-birth rates [( 23.3 to $52.0 \%(\mathrm{p}<0.05)]$, whereas women with fibroids not distorting the uterine cavity can achieve high live-birth rates without intervention. ${ }^{10}$

\section{INVESTIGATIONS AND WORK-UP}

Symptoms of myomas vary from abnormal uterine bleeding to pelvic pressure and/or pain, increasing abdominal girth, urinary or rectal symptoms, and reproductive failure. Examination may reveal increased size of uterus and irregularity in shape. Ultrasonography (USG) is the first-line investigation-number, size, and location of myomas may be determined on USG. Three-dimensional ultrasound can give valuable information about relationship of fibroids with endometrial cavity. ${ }^{1,3,4}$

Several publications have shown that ultrasound for detection of myomas is highly sensitive $(90-100 \%)$ and that it also has good specificity (87-98\%), positive predictive value (81-93\%), and negative predictive value (98-100\%). ${ }^{1,11-13}$ Magnetic resonance imaging (MRI) has gained popularity recently. However, it does not add clinically relevant information in most cases. ${ }^{1}$ USG appears to be as efficient as MRI in fibroid detection and essentially as good for assessing their size and location if the uterus has less than five lesions. ${ }^{14}$ Conversely, when the number of lesions is high, MRI exceeds ultrasound's technical limitation in precise fibroid mapping and characterization. ${ }^{4}$ The main sonographic markers for the differentiation of an adenomyoma from a fibroid are the absence of a lesion margin and the 
presence of lacunae. ${ }^{4}$ When doubts persist, however, MRI may reliably discriminate between the two conditions. ${ }^{4,15,16}$ Transvaginal ultrasound in conjunction with the introduction of sterile saline (sonohysterography) can determine whether a centrally located myoma impinges directly on the uterine cavity and may be amenable to treatment by hysteroscopic resection rather than by abdominal myomectomy. ${ }^{1}$

In infertile women with uterine myomas, thorough evaluation to exclude other common and possibly coexisting causes of infertility should be completed before concluding that a specific treatment of myomas is indicated. ${ }^{6}$

Hysterosalpingography (HSG) is indicated to assess the uterine cavity and tubal patency. Hysteroscopy can reveal any distortion of uterine cavity and gives valuable information whether submucosal fibroids can be removed surgically. Sonohysterography is highly sensitive for identifying intrau-terine lesions and yields results that correlate well with those obtained by hysteroscopy. ${ }^{17}$

Fibroids are traditionally classified according to their anatomical location and are divided in submucous, intramural, or subserosal locations. ${ }^{18}$ Bejakal and $\mathrm{Li}^{18}$ support the following definition: submucous fibroids are those that distort the uterine cavity and are further divided into three subtypes: pedunculated (type 0), sessile with intra-mural extension of fibroid $<50 \%$ (type I), ${ }^{19}$ and sessile with intramural extension $>50 \%$ (type II). Intramural fibroids are those which do not distort the cavity and with $50 \%$ of the tumor protruding into the serosal surface of the uterus. Fibroids protruding 50\% out of the serosal surface are considered subserosal. They are further divided into sessile or pedunculated. $^{18}$

\section{FIBROIDS AND INFERTILITY-EVIDENCE FROM LITERATURE}

The very fact that fibroids are more common in nulliparous compared with parous women indicates an association of fibroids with infertility. Several studies have investigated the relationship of fibroids with infertility. However, the results from these studies are not consistent. Most of the studies are underpowered and retrospective and there is no systematic controlled data for age and proper evaluation of endometrial cavity has not been performed in most of the studies. The relationship of fibroids with fertility has been studied in IVF-intracytoplasmic sperm injection (ICSI) cycles as well. There is an agreement that submucosal fibroids are detrimental, whereas subserosal lesions are not as far as fertility is concerned. The impact of intramural fibroids on reproduction is more controversial.

Though randomized studies are lacking, it has been shown that surgical treatment appears to increase the pregnancy rate: $50 \%$ women who undergo myomectomy for infertility, subsequently conceive. Available evidence also suggests that submucosal, intramural, and subserosal fibroids interfere with fertility in decreasing order of importance. ${ }^{4}$ Although more limited, some data supports an impact of the number and dimension of the lesions.

Somigliana et al, ${ }^{4}$ performed a meta-analysis of studies investigating the effect of fibroids located at different sites in IVF cycles. Based on their findings, it was concluded that myomas negatively affect pregnancy rates. The opinion given was that submucosal lesions appear to strongly interfere with the chance of pregnancy: the common OR $(95 \%$ $\mathrm{CI})$ for conception and delivery was 0.3 (0.1-0.7) and 0.3 (0.1-0.8), respectively. The impact of intramural myomas was shown to be less dramatic, the common OR $(95 \% \mathrm{CI})$ for conception and delivery was 0.8 (0.6-0.9) and 0.7 (0.5-0.8), respectively. Subserosal fibroids do not seem to play a role. ${ }^{4}$

Pritts et $\mathrm{al}^{20}$ published updated systematic review and meta-analysis of various studies addressing fibroids and infertility. A total of 23 studies were included-out of which one was randomized controlled trial, nine were prospective studies and rest were retrospective. The women with submucosal fibroids, compared with infertile women without fibroids, demonstrated a significantly lower clinical pregnancy rate [relative risk (RR) $0.363,95 \%$ CI 0.179-0.737], implantation rate $(0.283,95 \%$ CI $0.123-0.649)$, and ongoing pregnancy/live-birth rate $(0.31895 \%$ CI $0.119-0.850)$ and a significantly higher spontaneous abortion rate $(1.678,95 \%$ CI 1.373-2.051). No difference was seen in rate of preterm delivery. When women with subserosal fibroids were examined in comparison with women without fibroids, no difference was observed for any outcome measure. In contrast, presence of intramural fibroids produced significantly lower clinical pregnancy rates $(0.810,95 \%$ CI 0.696-0.941), implantation rates $(0.684,95 \%$ CI $0.587-0.796)$, and ongoing pregnancy/live-birth rates $(0.703,95 \%$ CI $0.583-0.848)$, and significantly higher spontaneous abortion rates $(1.747$, 95\% CI 1.226-2.489). The conclusions drawn from the meta-analysis were that fertility outcomes are decreased in women with submucosal fibroids and removal seems to confer benefit. Subserosal fibroids do not affect fertility outcomes and removal does not provide any benefit. Intramural fibroids appear to decrease fertility, but the results of therapy are unclear. $^{20}$

Sunkara et $\mathrm{al}^{21}$ conducted in a systematic review and meta-analysis on the most controversial issue of association between intramural fibroid not distorting the cavity on IVF outcome. A total of 19 observational studies comprising 6087 IVF cycles were evaluated. Meta-analysis of these studies showed a significant decrease in the live birth $(R R=0.79$, 95\% CI: 0.70-0.88, $\mathrm{p}<0.0001)$ and clinical pregnancy rate 
$(\mathrm{RR}=0.85,95 \%$ CI: 0.77-0.94, $\mathrm{p}<0.002)$ in women with noncavity-distorting intramural fibroids compared with those without fibroids, following IVF treatment. Inverse relationship between IVF outcome and the presence of noncavity distorting intramural fibroid may be explained by altered uterine vascular perfusion, myometrial contractility, endometrial function, gamete migration, or myometrial/ endometrial gene expression.

Contrary to this, in earlier study by Oliveira ${ }^{22}$ in a retrospective review of 247 women it was shown that patients having subserosal or intramural leiomyomas of $4 \mathrm{~cm}$ not encroaching on the uterine cavity have IVF-ICSI outcomes comparable to those of patients without such leiomyomas.

Somgliana, ${ }^{23}$ in a prospective cohort study failed to observe a detrimental effect of fibroids measuring upto $50 \mathrm{~mm}$ and not encroaching the uterine cavity on IVF outcome. There were 119 women in case (fibroids measuring upto $50 \mathrm{~mm}$ and not distorting the cavity as evaluated by hysterosonography) and control group (matched women free of fibroids). The adjusted OR for clinical pregnancy in affected women was 1.38 (95\% CI: 0.73-2.60). The number of deliveries was $22(18 \%)$ and $16(13 \%)$, respectively $(\mathrm{p}=0.38)$. The adjusted OR was $1.45(95 \% \mathrm{CI}$ : 0.71-2.94). Similar results emerged when focusing exclusively on women carrying intramural lesions ( $\mathrm{n}=80$ couples). There was no significant relationship between clinical outcome and either the number or size of the fibroids. ${ }^{23}$

Metwally et $\mathrm{a}^{24}$ further highlighted the importance of flaws in the existing literature regarding the effect of intramural fibroids, not distorting the cavity, on IVF outcome. After taking into consideration various confounding variables, the combined analysis of the 10 included studies, showed no evidence of a significant effect for intramural fibroids on clinical pregnancy rate (OR 0.74, 95\% CI 0.50-1.09), livebirth rate (OR $1.17,95 \% \mathrm{CI} 0.62-2.22)$, or miscarriage rate (OR 1.61, 95\% CI 0.61-4.20). There was also no evidence for a significant effect for myomectomy on the clinical pregnancy rate (OR $1.88,95 \%$ CI $0.57-6.14)$ or the miscarriage rate (OR $0.89,95 \%$ CI 0.14-5.48). Confounding variables which were taken into consideration were body mass index, smoking, age, location of fibroids, and cause of infertility. ${ }^{24}$

So looking at the above literature, it is clear that submucosal fibroids and large intramural ( $>5 \mathrm{~cm}$ in size) myomas distorting the endometrial cavity have detrimental effect on fertility and their removal is warranted before going for any kind of treatment for infertility or pregnancy loss. Subserosal myomas have least effect on fertility and their removal is not required for the treatment of fertility. Literature regarding management of small intramural fibroids, not distorting the uterine cavity, is controversial.

\section{CLINICAL MANAGEMENT OF MYOMAS}

\section{Expectant Management}

Small asymptomatic fibroids-intramural $(<5 \mathrm{~cm})$, not distorting the endometrial cavity and subserosal fibroids, in an otherwise infertile women can be treated by expectant management. In case of fibroids not distorting uterine cavity, surgery may be reserved for advanced cases, after repeated IVF failures or if fibroid-related obstetrical complications (i.e., recurrent miscarriage) have been observed in the recent past. But submucosal fibroids, even when small sized, need removal. ${ }^{1,3,4}$

\section{Medical Management}

Various medical modalities have been shown to cause shrinkage in size of myoma like GnRH analogs, mifepristone, danazol, gastrinone, estrogen, and progesterone modulators. But none of these improves fertility. Furthermore, there they can lead to a delay in more effective treatment for infertility. So none of these is recommended for treatment of infertility. The only indication for medical management may be-the use of $\mathrm{GnRH}$ analogs to decrease the size of fibroids before myomectomy. ${ }^{1,2,25}$

\section{Surgical Treatment}

Myomectomy is the mainstay of treatment for fibroids when child-bearing is a concern. Myomectomy can be done by laparotomy, laparoscopy, or hysteroscopy depending upon the size, number, and location of fibroids, expertize of surgeon and equipment available. When planning for myomectomy, patient should be thoroughly counseled regarding the procedure, its complications and likely outcome. Preoperative semen analysis, hysterosalpingography/hysteroscopy for evaluation of cavity and routine preoperative investigations should be done. ${ }^{1-3,25}$

Hysteroscopic myomectomy: Intracavitary and submucosal fibroids (when the major portion, i.e., at least $50 \%$ of fibroid is projecting into the uterine cavity), depending upon the size may be amenable to hysteroscopic resection, which should be carried out in a center with experience in handling the condition. Patients should be counseled about risk of uterine perforation and possible effects on pregnancy and fertility. Myomas may be removed using hysteroscopic scissors, monopolar or bipolar electrosurgical techniques, or mechanical morcellators or by laser methods. The procedure is difficult and challenging and complications include uterine perforation, hemorrhage, fluid overload, and hyponatremia (when distension media other than saline are used). The additional risk of postoperative intrauterine adhesions increases with the size and number of myomas and the extent of endometrial disruption. Measures commonly used in efforts 
to decrease the risk of postoperative adhesions include inserting a balloon catheter in the uterus to prevent apposition of denuded surfaces for approximately 1 week after surgery, treatment with high doses of estrogen to promote proliferation of the endometrium, and early 'second-look' hysteroscopy for lysis of recurrent adhesions. ${ }^{1,2,25}$ However, none of these techniques has been evaluated rigorously. There have been no randomized controlled trials aimed at assessing fertility after hysteroscopic myomectomy. In a small prospective cohort study, $72 \%$ of women with primary infertility conceived within 4 years after surgery and without further intervention; the miscarriage rate after surgery was $26 \%$ compared with $62 \%$ before hysteroscopic myomectomy for women having a previous miscarriage. ${ }^{26}$

Laparoscopic myomectomy: Laparoscopic myomectomy is suitable alternative to laparotomy in expert hands at specialized center. Patient might appreciate small but real risk of excessive hemorrhage during myomectomy which might necessitate hysterectomy. Main advantage with laparoscopy is shorter hospital stay, early recovery, and less chances of postoperative adhesion formation. Use of dilute vasopressin (10 units in $500 \mathrm{ml}$ of saline) during myomectomy has been shown to reduce blood loss during surgery, but its adverse effects on cardiovascular system should be kept in mind. ${ }^{27}$ Various studies have found no differences in operative time or blood loss but noted that laparoscopy was associated with less postoperative pain, a shorter length of stay, and a more rapid recovery. ${ }^{1,28,29}$ Cochrane review by Griffith et $\mathrm{al}^{30}$ concluded that there is limited evidence to suggest that there is any difference in fertility outcome if fibroids are removed via laparotomy when compared to laparoscopy.

Another laparoscopic technique, myolysis, involves thermal destruction of myomas via insertion of cryoprobes, electrocautery needles, or fiberoptic lasers. ${ }^{1,3}$ A nonsurgical method for myolysis involving MRI-guided focused ultrasonic treatment also has been described. ${ }^{1,31}$ Data relating to the short-and long-term outcomes achieved with such treatments are still lacking and, until they become available, myolysis cannot be recommended for women hoping to maintain or improve their fertility.

Open myomectomy: Open myomectomy has been the traditional technique for removal of fibroids. Main concern with open myomectomy has been the risk of operative blood loss and postoperative adhesion formation. ${ }^{1-3}$ Techniques which have been applied to reduce intraoperative blood loss during abdominal myomectomy include applying a tourniquet around the uterine isthmus to occlude the uterine vessels; placement of vascular clamps on the infundibulopelvic ligaments; transverse uterine incisions, parallel to myometrial vessels; and injection of dilute vasopressin into the overlying serosa and adjacent myometrium. ${ }^{32,33}$ The incidence of adhe- sions is extremely high (94\%) when myomectomy requires incisions on the posterior wall of the uterus and is lower, but still relatively high $(55 \%)$, when the anterior surface of the uterus is incised ${ }^{1,2,34}$ posterior pelvic adhesions also tend to be more dense and severe. Consequently, anterior uterine incisions are preferable, whenever possible. Postoperative adhesions that distort the adnexal anatomy may compromise future fertility.

Preoperative use of GnRH analogs: GnRH analogue use before surgery might be helpful to improve hemoglobin level in anemic patients when used along with hematinics. It is assumed to decrease operative blood loss but there is no definite data supporting this. First preoperative GnRH treatment leads to loss of planes leading to technical difficulty and might increase the operating time. Second, the recurrence rate/persistent after surgery is more with use of $\mathrm{GnRH}$ analogs. Some investigators have supported the idea of preoperative GnRH before hysteroscopic myomectomy suggesting that such treatment might facilitate surgery by cauing atrophy of adjacent tissue and by reducing the size of fibroids. ${ }^{1,3}$

Pregnancy rates after myomectomy: There is marked relief of symptoms after myomectomy in symptomatic women. Reported pregnancy rates after myomectomy are shown to be between 40 and $50 \%$. Pregnancy rate is not correlated to number or size of myomas removed and majority of pregnancies occur within first year after myomectomy. Recurrence rate of fibroids after myomectomy is up to 20 to $30 \%{ }^{1,3}$ Most of the studies evaluating the pregnancy outcome after myomectomy are retrospective.

Regarding the effect on miscarriage rate, a retrospective study observed that $19 \%$ of pregnancies ended in spontaneous abortion after surgery, compared with $41 \%$ before myomectomy. ${ }^{6}$

In a prospective controlled trial, Casini et $\mathrm{al}^{35}$ evaluated the effects of myomectomy on fertility. Casini et al randomized 181 women with a combination of submucosal, intramural, and subserosal fibroids to either surgery or expectant management and reported the pregnancy rates in each subgroup following timed intercourse. The authors found that myomectomy resulted in a statistically significant higher pregnancy rate among women with submucosal fibroids (43 vs 27\%), as well as those with a combination of submucosal and intramural fibroids (36 vs 15\%). There was a clinically significant trend toward better pregnancy rate in the group with only intramural fibroids after myomectomy (56\% vs 41\%), although the difference did not reach statistical significance.

In a updated systematic review by Pritts et al, regarding impact of myomectomy, when the control group was women with fibroids in situ, in those with submucosal fibroids, clinical pregnancy rate was higher in the myomectomy 
group, but the ongoing pregnancy/live-birth rate failed to reach statistical significance. The spontaneous abortion rate was unchanged. In women with intramural fibroids, no significant differences were seen. When the control group was the women with infertility without any fibroids, no statistically significant difference was seen in submucosal group. ${ }^{20}$ There are limited data regarding other obstetrical complications like preterm labor, malpresentations, and risk of scar rupture after myomectomy. Although it is generally recommended that women who undergo abdominal myomectomy should not be allowed to labor and should deliver by cesarean section, there is no direct evidence to support the recommendation. One study observed no uterine ruptures associated with 212 births after myomectomy, $83 \%$ of which were vaginal deliveries. 1,36

Based on these studies, it appears that myomectomy is beneficial and corrects the adverse reproductive outcome associated with submucosal and intramural fibroids.

Role of uterine artery embolization (UAE): Although uterine artery embolization has been shown to lead to marked improvement in symptoms related to fibroids, but this cannot be recommended for treatment of infertility. Adverse events following UAE have included infectious complications requiring hysterectomy, transcervical expulsion of a myoma, pelvic pain, nonpurulent vaginal discharge, delayed diagnosis of leiomyosarcoma, and diminished ovarian reserve or premature ovarian failure, particularly in older women. There is evidence to show increased risk of intrauterine adhesion formation after UAE. ${ }^{1,3,37}$ Successful pregnancies after UAE have been described, but data relating to fertility and pregnancy outcomes after UAE are still quite limited, and the procedure therefore cannot be recommended for women planning a later pregnancy.

Homer and Saridogan ${ }^{38}$ in a systematic review and meta-analysis of 227 completed pregnancies after UAE showed that miscarriage rates were higher in UAE pregnancies (35.2\%) compared with fibroid-containing pregnancies matched for age and fibroid location (16.5\%) (OR 2.8; 95\% CI 2.0-3.8). The UAE pregnancies were more likely to be delivered by cesarean section ( 66 vs $48.5 \%$; OR 2.1 ; $95 \% \mathrm{CI}$ 1.4-2.9) and to experience PPH \{13.9 vs 2.5\%; OR 6.4; $95 \%$ CI 3.5-11.7\}.${ }^{38}$ American College of Obstetricians and Gynecologists recommended that UAE should be considered investigational or relatively contraindicated in women wishing to retain fertility. ${ }^{39}$

But RCOG clinical recommendation on use of UAE states that in women who desire pregnancy but suffer from subfertility or recurrent miscarriage due to fibroids, who are unsuitable for hysteroscopic resection or myomectomy, or in whom myomectomy has failed can be offered UAE as safe effective alternative. ${ }^{25}$

\section{CONCLUSION}

Though myomas may not be a sole cause for infertility in majority of cases but fibroids that distort the uterine cavity and large intramural fibroids are shown to be associated with adverse effects on reproductive function. In infertile women and those with recurrent pregnancy loss, myomectomy should be considered only after a thorough evaluation has been completed. Medical management of fibroids has no role in treatment of fertility rather it might lead to delay in the final treatment for infertility. Preoperative medical treatment with a GnRH agonist should be considered for women who are anemic and those undergoing hysteroscopic myomectomy. Subserosal fibroids have least effect on fertility, so they do not need removal before infertility treatment. UAE or MRI-guided ultrasonic treatment is not recommended where fertility is a concern.

\section{REFERENCES}

1. Practice Committee, American Society for Reproductive Medicine. ACOG Education Bulletin. Myomas and Reproduction. Fertil and Steril 2008, 90, Suppl 3:S125-S130.

2. Letterie GS. Leiomyomas, fertility and ART. Surgery, assisted reproductive technology and infertility. 2nd ed. 2005 Taylor and Francis, Abingdon, Oxon.

3. Breech LL, Rock JA. Leiomyomata uterus and myomectomy. Chapter 31, Te Linde's operative gynaecology. 10th ed. Lippincott Williams and Wilkins 2008.

4. Somigliana E, Vercellini P, Daguati R, Pasin R, Giorgi OG, Crosignani PG. Fibroids and female reproduction: a critical analysis of the evidence. Hum Reprod Update 2007;13(5): 465-476.

5. Ben-Nagi J, Miell J, Mavrelos D, Naftalin J, Lee C, Jurkovic D. Endometrial implantation factors in women with submucosal fibroids. RBM Online 2010;21:610-615.

6. Buttram VC Jr, Reiter RC. Uterine leiomyomata: etiology, symptomatology and management. Fertil Steril 1981;36:433-435.

7. Klatsky PC, Tran ND, Caughey AB, Fujimoto VY. Fibroids and reproductive outcomes: a systematic literature review from conception to delivery. Am J Obstet Gynecol 2008;198:357-366.

8. RiceJP, KayHH, MahonyBS. The clinical significance of uterine leiomyomas in pregnancy. Am J Obstet Gynecol 1989;160:1212-1216.

9. Muram D, Gillieson M, Walters JH. Myomas of the uterus in pregnancy: ultrasonographic follow-up. Am J Obstet Gynecol 1980;138:16-19.

10. Saravelos SH, Yan J, Rehmani H, Li TC. The prevalence and impact of fibroids and their treatment on the outcome of pregnancy in women with recurrent miscarriage. Hum Rep 2011;26(12): 3274-3279.

11. Cicinelli E, Romano F, Anastasio PS, Blasi N, Parisi C, Galantino P. Transabdominal sonohysterography, transvaginal sonography, and hysteroscopy in the evaluation of submucous myomas. Obstet Gynecol 1995;85:42-47.

12. Indman PD. Abnormal uterine bleeding. Accuracy of vaginal probe ultrasound in predicting abnormal hysteroscopic findings. J Reprod Med 1995;40:545-548.

13. Becker E Jr, Lev-Toaff AS, Kaufman EP, Halpern EJ, Edelweiss MI, Kurtz AB. The added value of transvaginal sonohysterography over transvaginal sonography alone in women with known or suspected leiomyoma. J Ultrasound Med 2002;21:237-247. 
14. Dueholm M, Lundorf E, Hansen ES, Ledertoug S, Olesen F. Accuracy of magnetic resonance imaging and transvaginal ultrasonography in the diagnosis, mapping, and measurement of uterine myomas. Am J Obstet Gynecol 2002;186:409-415.

15. Farquhar C, Brosens I. Medical and surgical management of adenomyosis. Best Pract Res Clin Obstet Gynaecol 2006;20:603-616.

16. Tamai K, Koyama T, Umeoka S, Saga T, Fujii S, Togashi K. Spectrum of MR features in adenomyosis. Best Pract Res Clin Obstet Gynaecol 2006;20:583-602.

17. Goldberg J, Falcone T, Attaran M. Sonohysteroscopic evaluation of uterine defects noted on hysterosalpingography. Hum Reprod 1997;12:2115-2117.

18. Bajekal N, Li TC. Fibroids, infertility and pregnancy wastage. Hum Reprod Update 2000;6:614-662.

19. Wamsteker K, Emanuel MH, de Kruif JH. Transcervical hysteroscopic resection of submucous fibroids for abnormal uterine bleeding: results regarding the degree of intramural extension. Obstet Gynecol 1993;82:736-740.

20. Pritts EA, Parker WA, Olive DL. Fibroids and infertility: an updated systematic review of the evidence. Fertil and Steril 2009; 91(4):1215-1223.

21. Sunkara SK, Khairy M, El-Toukhy T, KhalafY, Coomarasamy A. The effect of intramural fibroids without uterine cavity involvement on the outcome of IVF treatment: a systematic review and meta-analysis. Hum Reprod 2010;25(2):418-442.

22. Oliveira FG, Abdelmassih VG, Diamond MP, Dozortsev D, Melo NR, Abdelmassih R. Impact of subserosal and intramural uterine fibroids that do not distort the endometrial cavity on the outcome of in vitro fertilization-intracytoplasmic sperm injection. Fertil and Steril 2004,8(3):582-587.

23. Somigliana E, Benedictis SD, Vercellini P, Nicolosi AE, Benaglia L, Scarduelli C, Ragni G, Fedele L. Fibroids not encroaching the endometrial cavity and IVF success rate: a prospective study. Hum Reprod 2011;26(4):834-839.

24. Metwally M, Farquhar CM, Li TC. Is another meta-analysis on the effects of intramural fibroids on reproductive outcomes needed? Reprod BioMed Online 2011;23:2-14.

25 . Royal college of radiologists and royal college of obstetricians and gynaecologists. Clinical recommendations on the use of UAE in the management of fibroids. 2nd ed. London. The Royal College of Radiologists, 2009;p10.
26. Shokeir TA. Hysteroscopic management in submucous fibroids to improve fertility. Arch Gynecol Obstet 2005;273:50-54.

27. Sizzi O, Rossetti A, Malzoni M, et al. Italian multicentre study on complications of laparoscopic myomectomy. J Minim Invasive Gynecol 2007;14:453-462.

28. Mais V, Ajossa S, Guerriero S, Mascia M, Solla E, Melis GB. Laparoscopic versus abdominal myomectomy: a prospective, randomized trial to evaluate benefits in early outcome. Am J Obstet Gynecol 1996;174: 654-658.

29. Seracchioli R, Rossi S, Govoni F, Rossi E, Venturoli S, Bulletti C, et al. Fertility and obstetric outcome after laparoscopic myomectomy of large myomata: a randomized comparison with abdominal myomectomy. Hum Reprod 2000;15:2663-2668.

30. Griffiths AN, D'Angelo A, Amso NN. Surgical treatment of fibroids for subfertility. Cochrane Database of Systematic Reviews 2006;(1).

31. Zupi E, Sbracia M, Marconi D, Munro MG. Myolysis of uterine fibroids: is there a role? Clin Obstet Gynecol 2006;49:821-833.

32. Ginsburg ES, Benson CB, Garfield JM, Gleason RE, Friedman AJ. The effect of operative technique and uterine size on blood loss during myomectomy: a prospective randomized study. Fertil Steril 1993;60:956-962.

33. Frederick J, Fletcher H, Simeon D, Mullings A, Hardie M. Intramyometrial vasopressin as a haemostatic agent during myomectomy. Br J Obstet Gynaecol 1994;101:435-437.

34. Tulandi T, Murray C, Guralnick M. Adhesion formation and reproductive outcome after myomectomy and second-look laparoscopy. Obstet Gynecol 1993;82:213-215.

35. Casini ML, Rossi F, Agostini R, Unfer V. Effect of the position of fibroids on fertility. Gynecol. Endocrinol 2006;22:106-109.

36. Davids A. Myomectomy: surgical technique and results in a series of 1,150 cases. Am J Obstet Gynecol 1952;63:592-604.

37. Mara M, Fucikova Z, Kuzel D, Maskova J, Dundr P, Zizka Z. Hysteroscopy after uterine fibroid embolization in women of fertile age. J Obstet Gynaecol Res 2007;33:316-324.

38. Homer H, Saridogan E. Uterine artery embolization for fibroids is associated with an increased risk of miscarriage. Fertil and Steril 2010;94(1):324-330.

39. Committee on Gynecologic Practice ACOG. American College of Obstetricians and Gynecologists (ACOG) Committee Opinion. Uterine artery embolization. Obstet Gynecol 2004;103:403-404. 